\title{
A case report of eyelid Merkel cell carcinoma occurring under treatment with nivolumab for a lung adenocarcinoma
}

Daniele Lavacchi ${ }^{1}$, Stefania Nobili ${ }^{*}{ }^{*}$, Marco Brugia ${ }^{3}$, Agnese Paderi $^{1}$, Sara Fancelli ${ }^{1}$, Enrico Caliman ${ }^{1}$, Federica Vergoni ${ }^{4}$ and Enrico Mini ${ }^{2^{*}}$

\begin{abstract}
Background: Merkel cell carcinoma (MCC) is a rare neuroendocrine malignancy of the skin characterized by high aggressiveness. Four main factors are implicated in its development: immunosuppression, ultraviolet radiation, age and the Merkel cell polyomavirus (MCPyV). In recent years, immune checkpoint inhibitors have shown clinical activity in MCC treatment.

Case presentation: We report the case of an 82-year-old man with a lung adenocarcinoma diagnosis, who underwent immunotherapy with nivolumab as second-line treatment. Seven months after the diagnosis of lung cancer during the nivolumab treatment, the patient developed an eyelid MCC, initially misdiagnosed as a chalazion. A palliative radiotherapy was performed with clinical benefit. After a total of seven cycles of nivolumab, computed tomography showed a lung and cerebral disease progression. In addition, clinical conditions worsened leading to the patient's death 13 months after the initial lung cancer diagnosis.

Conclusions: Cases of co-occurrence of MCC and non-small cell lung cancer (NSCLC) have rarely been reported. Interestingly, common risk factors may be postulated for both cancers. Considering the rarity of this adverse event, its short-term temporal relation with the administration of the drug, which makes a relation improbable, and the coexistence of other risk factors, which may provide plausible explanations, it is possible to conclude according to the WHO Adverse Reaction Terminology that a causal relation between the occurrence of this serious adverse event and the exposure to the drug is unlikely. However, the case deserves to be reported in the literature.
\end{abstract}

Keywords: Merkel cell carcinoma, NSCLC, Nivolumab, MCPyV, Eyelid MCC, Elderly

\section{Background}

Merkel cell carcinoma (MCC) is a rare neuroendocrine malignancy of the skin characterized by a high aggressiveness with an overall survival of 10 months in the metastatic setting $[1,2]$. It is an age-related cancer with a higher incidence in elderly patients. Historically, the two main factors implicated in the onset of MCC were exposure to ultraviolet rays and immunosuppression [3]. In 2008 Feng et al. revealed a new polyomavirus, until then unknown, in MCC tissue samples - the Merkel cell polyomavirus (MCPyV) [4]. Several studies have shown

\footnotetext{
*Correspondence: stefania.nobili@unifi.it; enrico.mini@unifi.it ${ }^{2}$ Department of Health Sciences, University of Florence, viale Pieraccini, 6 , 50139 Florence, Italy

Full list of author information is available at the end of the article
}

that MCPyV-DNA is integrated into tumor cells in about $80 \%$ of MCC cases, inferring that infection plays an important role in the pathogenesis of $\mathrm{MCC}[4,5]$.

About a half of all MCCs originate from the head and neck $(\mathrm{H} \& \mathrm{~N})$ area. $5-20 \%$ of the $\mathrm{H} \& \mathrm{~N}$ MCCs originate in the eyelids [6]. In most cases, the lesion is rapidly evolving and the diagnosis is not always readily identified. In fact, the lesion is often misdiagnosed as a chalazion or a stye $[6,7]$.

MCC shows very low response rates to cytotoxic chemotherapy [8-10]. In recent years, immune checkpoint inhibitors such as avelumab, an anti-programmed death ligand 1 (PD-L1) monoclonal antibody (MoAb), pembrolizumab and nivolumab, anti-programmed death 1 (PD-1) MoAbs, have shown clinical activity in the treatment of MCC. [11-16]. On March 23, 2017, the

(c) The Author(s). 2018 Open Access This article is distributed under the terms of the Creative Commons Attribution 4.0 International License (http://creativecommons.org/licenses/by/4.0/) which permits unrestricted use, distribution, and reproduction in any medium, provided you give appropriate credit to the original author(s) and the source, provide a link to the Creative Commons license, and indicate if changes were made. The Creative Commons Public Domain Dedication waiver (http://creativecommons.org/publicdomain/zero/1.0/) applies to the data made available in this article, unless otherwise stated. 
U.S. Food and Drug Administration granted accelerated approval to avelumab for the treatment of patients with metastatic MCC [11].

\section{Case presentation}

We report the case of an 82-year-old man, who underwent a total body computed tomography (CT) on February 2017, due to the occurrence of cough. CT showed an extensive mass in the left upper lobe of the lung. Thus, a bronchoscopy with transbronchial needle aspiration (TBNA) was performed. The cytological examination was compatible with lung adenocarcinoma. Epidermal growth factor receptor (EGFR) mutations and anaplastic lymphoma kinase (ALK) translocation were tested to determine the most appropriate treatment but no mutation was detected. It was not possible to test PD-L1 expression because only cytological samples were available.

To complete the staging of the disease, the patient underwent a positron emission tomography (PET) examination. PET showed a massive tracer uptake at the pulmonary mass and showed an extensive involvement of the hilar and mediastinal lymph nodes. Before starting the treatment, a further TC scan was performed in May. TC showed an increased pulmonary mass involving approximately the entire left lung.

The patient referred a smoking history and as comorbidities: arterial hypertension, osteoporotic and traumatic vertebral fractures, iatrogenic bone marrow lesion resulting from surgery for discopathy, benign prostatic hypertrophy treated with transurethral resection, pulmonary emphysema, carotid vasculopathy and abdominal aneurysm. He had an ECOG performance status of 2.

In relation to clinical conditions, age and comorbidities, the patient underwent two chemotherapy cycles with oral vinorelbine (day 1,8 every 21), the latter of which was administered in July. During the treatment, the patient experienced fatigue G1, diarrhea G1, constipation G1, anorexia G1 and hyperkinetic supraventricular arrhythmia treated with amiodarone.

The restaging CT was performed in July and showed lung disease progression. Thus, from July to December, the patient received $3 \mathrm{mg} / \mathrm{kg}$ nivolumab (day 1 every 14) as second-line treatment for a total of seven cycles. Based on body weight, nivolumab was administered at a dose of $195 \mathrm{mg}$ for the first two cycles and $205 \mathrm{mg}$ for the subsequent five cycles. After the first two doses, the patient was hospitalized at the emergency department with a diagnosis of pneumonia. Therefore, antibiotics and corticosteroids were administered and a clinical improvement was obtained. In September, the patient recovered a good respiratory performance, so he was able to restart nivolumab. The subsequent five cycles were well tolerated except for grade 1 hypothyroidism and grade 2 fatigue.
Collaterally, in September, the patient showed a small nodular dome shaped lesion on the upper eyelid. $\mathrm{He}$ consulted a private ophthalmologist who initially suspected a diagnosis of chalazion. However, the lesion increased until about $3 \mathrm{~cm}$ within a few weeks. Thus, the patient came back to the Ophthalmology Department of Azienda Ospedaliera-Universitaria Careggi of Florence where a biopsy was performed, with the suspect of a malignant lesion. The histological examination indicated an MCC (immunohistochemical study: cytokeratin 20+, synaptophysin+, chromogranin+, CD20-, CD3-, Ki67 60-70\%) (Fig. 1).

A nuclear magnetic resonance imaging of the orbit and neck did not show lymph node involvement.

The case was collegially discussed. Since the patient was at high risk for anesthesia due to his clinical conditions and given the prognosis of lung cancer, surgery was contraindicated. Thus, a palliative radiotherapy was proposed and was started in December when the lesion had expanded to about $5 \mathrm{~cm}$ in the anteroposterior diameter. Following radiotherapy, the size of the eyelid lesion was reduced to a few millimeters.

In December 2017, after a total of seven cycles of nivolumab, a CT scan was also performed. It showed a lung and cerebral disease progression. In addition, there was a worsening of clinical conditions with increased cough and fatigue. Therefore, the best supportive care was provided until the patient died in March 2018.

\section{Discussion and conclusions}

Our patient developed an eyelid MCC 7 months after the diagnosis of lung cancer. Available data in literature shows that, overall, there is no statistically significant risk to develop an MCC following a diagnosis of primary lung cancer (i.e. standardized significant rate (SIR) 0.88; 95\% CI 0.32-1.92), as well as to develop an MCC within 1 year from the diagnosis of lung cancer ((SIR) 0.63; 95\% CI 0.13-1.85) [17].

The development of MCC occurred during a treatment with nivolumab for a lung adenocarcinoma. Nivolumab is a fully human IgG4 immune checkpoint inhibitor antibody targeting PD-1 that has been approved for the treatment of advanced or metastatic non-squamous non-small-cell lung cancer (NSCLC) according to positive results of the phase III, open label study CheckMate 057 [18].

An analysis of the literature was performed to know whether any MCC occurrences in the course of a nivolumab treatment had been reported. The research did not identify any publications related to the occurrence of MMC during nivolumab treatments. Based on post-marketing reports of the nivolumab producer, one case of neuroendocrine carcinoma during the treatment with this drug for lung adenocarcinoma has been described. However, since 


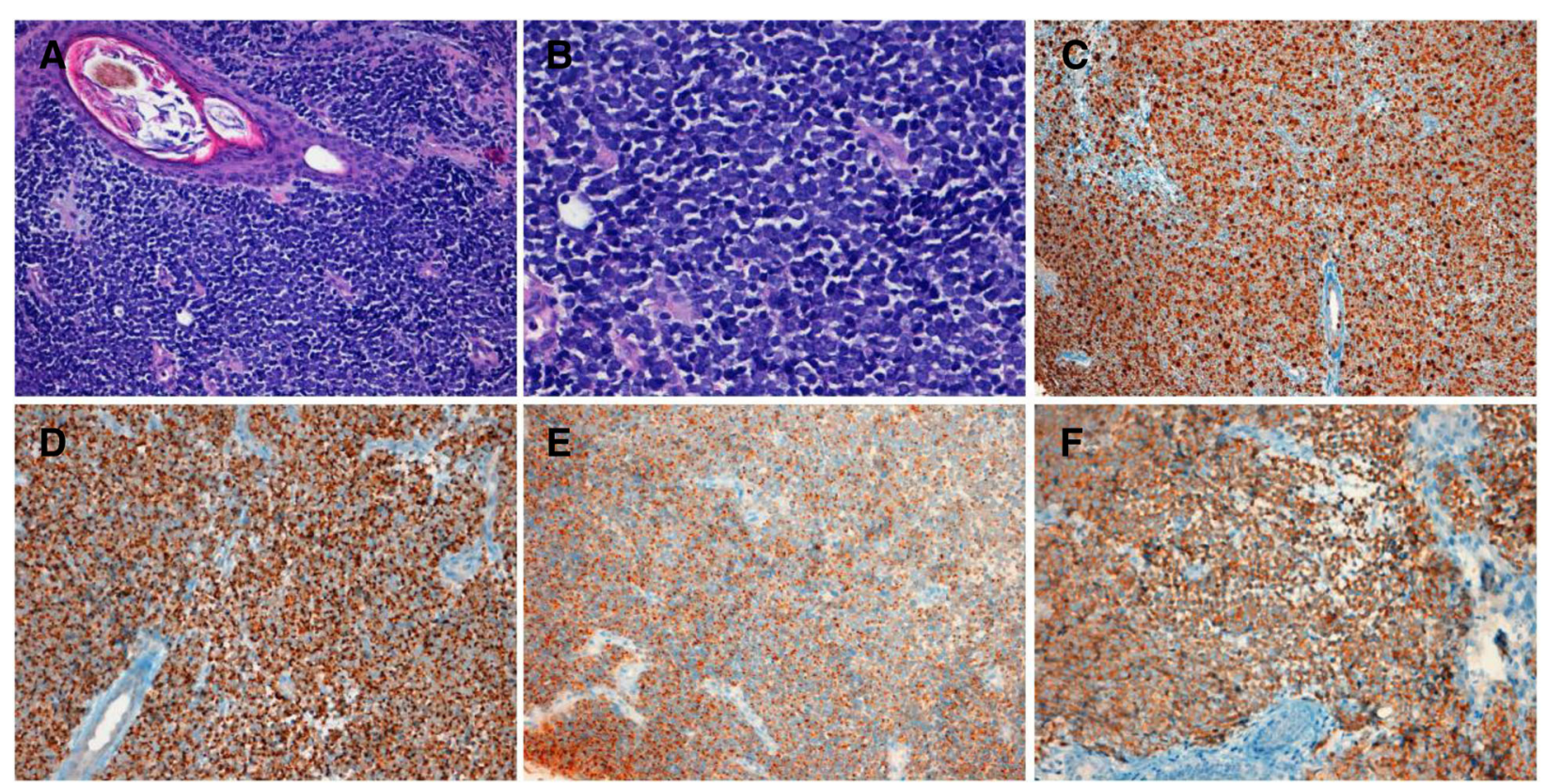

Fig. 1 Histological examination. a Hematoxylin-eosin (20x); b Hematoxylin-eosin (40x); c Ki67 (10x); d Cytokeratin 20 (20x); e Chromogranin (20x); f Synaptophysin (20x)

this type of event is reported voluntarily from a population of uncertain size, it is generally not possible to reliably estimate the frequency or to establish a causal relationship to the drug exposure.

MCC represents a checkpoint inhibitor responsive cancer. Factors that have been suggested to contribute to an immunotherapy response are represented by a high tumor mutational burden due to the ultraviolet rays exposure [19] and the integration of MCPyV DNA sequence. In fact, high expression levels of inhibitory receptors such as PD-1 and Tim-3 have been found on MCC infiltrating lymphocytes and $\mathrm{T}$ CD8-specific MCPyV cells [5]. These factors offer a rationale for treating patients with anti PD-1/anti PD-L1 monoclonal antibodies.

Avelumab is indicated in the treatment of MCC based on an open-label, single-arm, multi-center clinical trial (JAVELIN Merkel 200 trial). It induced an objective response (OR) in 33\% of treated patients [11]. Responses to avelumab were observed irrespective of PD-L1 expression on tumor cells or Merkel cell polyomavirus status [12].

In a multicentre phase 2 noncontrolled trial including 26 patients with an advanced MCC pembrolizumab induced an OR in $56 \%$ of patients. PD-L1 expression was more frequent in MCPyV-positive cancers than in MCPyV-negative cancers ( $71 \%$ vs $25 \%$ ). Responses were observed in both virus-positive and virus-negative. No significant correlation of PD-L1 expression on tumor cells or infiltrating immune cells with drug response was observed [13].
A significant and durable response in a patient with metastatic MCC following nivolumab therapy was reported [14]. Preliminary results of an ongoing noncomparative, multiple cohort, open-label, phase $1 / 2$ study (CheckMate 358$)$ of nivolumab in patients $(n=50$ MCC) with virus-positive and virus-negative solid tumors show an OR in $64 \%$ of advanced MCC cases and pathologic tumor regressions in $65 \%$ of resectable MCC cases $[15,16]$. Moreover, a phase 2 clinical trial evaluating nivolumab + ipilimumab $+/$ - stereotactic body radiation therapy (SBRT) for metastatic MCC is ongoing (NCT03071406).

Four main factors are implicated in the development of MCC: immunosuppression [20-25], ultraviolet radiation [3], age [26], and MCPyV [4, 5]. Overall, our patient presented three out of four MCC risk factors, whereas the MCPyV positivity status was unknown.

As far as immunosuppression is concerned, it is known that immunosuppressed patients have a poor prognosis with a reduced MCC-specific survival [20]. Immunosuppressive conditions determining an increased risk of developing an MCC are: patients with haematological malignancies, especially chronic lymphocytic leukaemia [21, 22], acquired immunodeficiency syndrome (AIDS) [23], patients undergoing organ transplantation [24] or patients in chronic treatment for autoimmune diseases [25]. Our patient did not have an immunosuppressive state such as those described above, but he had a lung cancer and he previously had received chemotherapy with vinorelbine. 
It is also well known that there is a strong association between MCC and a history of sun exposure [3]. Ultraviolet-associated mutations in RB1, TP53, NOTCH1 and FAT1 genes have been reported in MCPyV-negative cancers [19]. In our patient, the MCC developed in one of the most sun-exposed anatomical sites, so prolonged exposure to ultraviolet light may have been a relevant causal factor of the MCC. On the basis of the information gained by the anamnestic collection, a history of high sun exposure both before and during the treatments was highlighted.

Regarding age, it has been shown that H\&N MCCs have a higher incidence in elderly patients. It is known that MCPyV seroprevalence increases according to age achieving $73 \%$ in subjects older than 70 years [26].

Our patient had two cancers in which MCPyV has been proposed to be related with the pathogenesis. In fact, several studies have reported the presence of MCPyV-DNA integrated into tumor cells in about $80 \%$ of MCC cases $[4,5]$ and in a range from 9 to $18 \%$ in NSCLCs [27-29]. Thus, although the underlying tumorigenic mechanisms are unknown, in a subset of patients MCPyV could play an important role also in the pathogenesis of NSCLC, through the expression of two putagenic oncoproteins (i.e. large $\mathrm{T}$ and small $\mathrm{T}$ antigens) $[29,30]$. Unfortunately, we were not able to determine the presence of MCPyV DNA due to the unavailability of biological material. However, it must be noted that although $\mathrm{MCC}$ is a rare cancer, $\mathrm{MCPyV}$ is ubiquitous in human populations (i.e. from 60 to $80 \%$ of the general population is infected with MCPyV) [30].

In conclusion, we described a case of eyelid MCC occurring in a patient with recent diagnosis of NSCLC during second-line treatment with nivolumab. Cases of co-occurrence of MCC and NSCLC have rarely been reported. Interestingly, common risk factors may be postulated for both cancers. Considering the rarity of this adverse event, its short-term temporal relation with the administration of the drug, which makes a relation improbable, and the coexistence of other risk factors, which may provide plausible explanations, it is possible to conclude according to the WHO Adverse Reaction Terminology [31] that a causal relation between the occurrence of this serious adverse event and the exposure to the drug is unlikely. However, the case deserves to be reported in the literature.

\footnotetext{
Abbreviations

AIDS: Acquired immunodeficiency syndrome; ALK: Anaplastic lymphoma kinase; CT: Computed tomography; EGFR: Epidermal growth factor receptor; H\&N: Head and neck; MCC: Merkel cell carcinoma; MCPyV: Merkel cell polyomavirus; MoAb: Monoclonal antibody; NSCLC: Non-small-cell lung cancer; OR: Objective response; PD-1: programmed death 1; PDL1: Programmed death ligand 1; PET: Positron emission tomography; SBRT: Stereotactic body radiation therapy; SIR: Standardized significant rate; TBNA: transbronchial needle aspiration; WHO: World health organization
}

Acknowledgements

Not applicable.

Funding

No financial support was requested.

Availability of data and materials

All data generated or analyzed during this study are included in this published article.

\section{Authors' contributions}

EM, DL and SN wrote the manuscript and participated in the collection of clinical data and data analysis and clinical data interpretation. $E M, D L, M B$, AP, SF and EC contributed to the management of the clinical case and interpretation of clinical data. FV contributed to pathological examinations, and immunohistochemical analysis. All authors have read and approved the final manuscript.

Ethics approval and consent to participate

Not applicable

\section{Consent for publication}

Written informed consent was obtained from the patient's family for publication of this case report and any accompanying images.

Competing interests

The authors declare that they have no competing interests.

\section{Publisher's Note}

Springer Nature remains neutral with regard to jurisdictional claims in published maps and institutional affiliations.

\section{Author details \\ ${ }^{1}$ School of Human Health Sciences, University of Florence, Largo Brambilla 3, 50134 Florence, Italy. ${ }^{2}$ Department of Health Sciences, University of Florence, viale Pieraccini, 6, 50139 Florence, Italy. ${ }^{3}$ Department of Experimental and Clinical Medicine, University of Florence, Largo Brambilla 3, 50134 Florence, Italy. ${ }^{4}$ Pathological Anatomy Unit, Careggi University Hospital, Largo Brambilla 3, 50134 Florence, Italy.}

Received: 3 July 2018 Accepted: 9 October 2018

Published online: 22 October 2018

\section{References}

1. Harms $K L$, Healy MA, Nghiem $P$, et al. Analysis of prognostic factors from 9387 Merkel cell carcinoma cases forms the basis for the new 8th edition AJCC staging system. Ann Surg Oncol. 2016;23:3564-71.

2. Schadendorf D, Lebbé C, Zur Hausen A, et al. Merkel cell carcinoma: Epidemiology, prognosis, therapy and unmet medical needs. Eur J Cancer. 2016;71:53-69.

3. Heath M, Jaimes $N$, Lemos $B$, et al. Clinical characteristics of Merkel cell carcinoma at diagnosis in 195 patients: the "AEIOU" features. J Am Acad Dermatol. 2008;58(3):375-81.

4. Feng $H$, Shuda $M$, Chang $Y$, et al. Clonal integration of a polyomavirus in human Merkel cell carcinoma. Science. 2008;319(5866):1096-100.

5. Afanasiev OK, Yelistratova L, Miller N, et al. Merkel polyomavirus-specific T cells fluctuate with Merkel cell carcinoma burden and express therapeutically targetable PD-1 and Tim-3 exhaustion markers. Clin Cancer Res. 2013;19(19):5351-60

6. Merritt H, Sniegowski MC, Esmaeli B. Merkel cell carcinoma of the eyelid and periocular region. Cancers. 2014;6(2):1128-37.

7. Rubsamen PE, Tanenbaum M, Grove AS, et al. Merkel cell carcinoma of the eyelid and periocular tissues. Am J Ophthalmol. 1992;113(6):674-80.

8. Pectasides D, Pectasides M, Psyrri A, et al. Cisplatin-based chemotherapy for Merkel cell carcinoma of the skin. Cancer Investig. 2006:24(8):780-5.

9. National Comprehensive Cancer Network (NCCN). NCCN clinical practice guidelines in oncology. Version 1.2018.

10. Tai PT, Yu E, Winquist E, et al. Chemotherapy in neuroendocrine/Merkel cell carcinoma of the skin: case series and review of 204 cases. J Clin Oncol. 2000;18(12):2493-9. 
11. Kaufman HL, Russell J, Hamid O, et al. Avelumab in patients with chemotherapy-refractory metastatic Merkel cell carcinoma: a multicentre, single-group, open-label, phase 2 trial. Lancet Oncol. 2016;17(10):1374-85.

12. Kaufman $\mathrm{HL}$, Russell J, Hamid O, et al. Updated efficacy of avelumab in patients with previously treated metastatic Merkel cell carcinoma after $\geq 1$ year of follow-up: JAVELIN Merkel 200, a phase 2 clinical trial. J Immunother Cancer. 2018;6(1):7.

13. Nghiem PT, Bhatia S, Lipson EJ, et al. PD-1 blockade with Pembrolizumab in advanced Merkel-cell carcinoma. New Engl J Med. 2016;374(26):2542-52.

14. Walocko FM, Scheier BY, Harms PW, et al. Metastatic Merkel cell carcinoma response to nivolumab. J Immunother Cancer. 2016;4:79.

15. Topalian SL, Bhatia S, Hollebecque A, et al. Abstract CT074: noncomparative, open-label, multiple cohort, phase $1 / 2$ study to evaluate nivolumab (NIVO) in patients with virus-associated tumors (CheckMate 358): efficacy and safety in Merkel cell carcinoma (MCC). Am Assoc Cancer Res. 2017;77(13 Supplement):CT074.

16. Topalian SL, Bhatia S, Kudchadkar RR, et al. Nivolumab (Nivo) as neoadjuvant therapy in patients with resectable Merkel cell carcinoma (MCC) in CheckMate 358. J Clin Oncol 2018; 36 (suppl; abstr 9505).

17. Howard RA, Dores GM, Curtis RE, et al. Merkel cell carcinoma and multiple primary cancers. Cancer Epidemiol Biomark Prev. 2006;15(8):1545-9.

18. Borghaei H, Paz-Ares L, Horn L, et al. Nivolumab versus docetaxel in advanced nonsquamous non-small-cell lung cancer. N Engl J Med. 2015; 373:1627-39.

19. Wong SQ, Waldeck K, Vergara IA, Schröder J, Madore J, Wilmott JS, et al. UVassociated mutations underlie the etiology of MCV-negative Merkel cell carcinomas. Cancer Res. 2015;75:5228-34.

20. Paulson KG, lyer JG, Blom A, et al. Systemic immune suppression as a stageindependent predictor of diminished Merkel cell carcinoma-specific survival. J Invest Dermatol. 2013;133(3):642-6.

21. Brewer JD, Shanafelt TD, Otley CC, et al. Chronic lymphocytic leukemia is associated with decreased survival of patients with malignant melanoma and Merkel cell carcinoma in a SEER population-based study. J Clin Oncol. 2012;30:843-9

22. Quaglino D, Di Leonardo G, Lalli G, et al. Association between chronic lymphocytic leukaemia and secondary tumours: unusual occurrence of a neuroendocrine (Merkel cell) carcinoma. Eur Rev Med Pharmacol Sci. 1997;1:11-6

23. Engels EA, Frisch M, Goedert JJ, et al. Merkel cell carcinoma and HIV infection. Lancet. 2002;359:497-8.

24. Penn I, First MR. Merkel's cell carcinoma in organ recipients: report of 41 cases. Transplantation. 1999;68:1717-21.

25. Hemminki K, Liu X, Ji J, et al. Kaposi sarcoma and Merkel cell carcinoma after autoimmune disease. Int J Cancer. 2012;131(3):E326-8.

26. Viscidi RP, Rollison DE, Sondak VK, et al. Age-specific Seroprevalence of Merkel cell polyomavirus, BK virus, and JC virus. Clin Vaccine Immunol. 2011; 18(10):1737-43.

27. Lasithiotaki I, Antoniou KM, Derdas SP, et al. The presence of Merkel cell polyomavirus is associated with deregulated expression of BRAF and $\mathrm{BCl}-2$ genes in non-small cell lung cancer. Int J Cancer. 2013;133:604-11.

28. Hashida Y, Daibata M. Considerations on the link between Merkel cell polyomavirus and lung cancer. Lung Cancer Management 2014;3(4). https:// doi.org/10.2217//mt.14.20

29. Kim GJ, Lee J-H, Lee DH. Clinical and prognostic significance of Merkel cell polyomavirus in non small cell lung cancer. Medicine. 2017;96(3):e5413. https://doi.org/10.1097/MD.0000000000005413.

30. Coggshall K, Tello TL, North JP, et al. Merkel cell carcinoma: an update and review: pathogenesis, diagnosis, and staging. J Am Acad Dermatol. 2018; 78(3):433-42.

31. Edwards IR, Aronson JK. Adverse drug reactions: definitions, diagnosis, and management. Lancet. 2000:356(9237):1255-9.

\section{Ready to submit your research? Choose BMC and benefit from:}

- fast, convenient online submission

- thorough peer review by experienced researchers in your field

- rapid publication on acceptance

- support for research data, including large and complex data types

- gold Open Access which fosters wider collaboration and increased citations

- maximum visibility for your research: over $100 \mathrm{M}$ website views per year

At BMC, research is always in progress.

Learn more biomedcentral.com/submissions 